\title{
Spacetime is a quantum computer
}

\author{
Open Quantum Collaboration*† \\ July 22, 2020
}

\begin{abstract}
If spacetime is a quantum computer, then all physical systems are equivalent to quantum computational algorithms.
\end{abstract}

keywords: quantum spacetime, quantum computation, entanglement, quantum gravity

The most updated version of this paper is available at https://osf.io/rnckw/download

\section{Introduction}

1. Quantum theory operates in matter and energy.

2. Spacetime is entangled with matter/energy.

3. Due to (1) and (2), the quantum theory also rules spacetime.

\section{Conjecture}

4. Spacetime itself operates according to quantum computational algorithms.

*All authors with their affiliations appear at the end of this paper.

†Corresponding author: mplobo@uft.edu.br | Join the Open Quantum Collaboration 


\section{Overview}

5. $[1,2]$

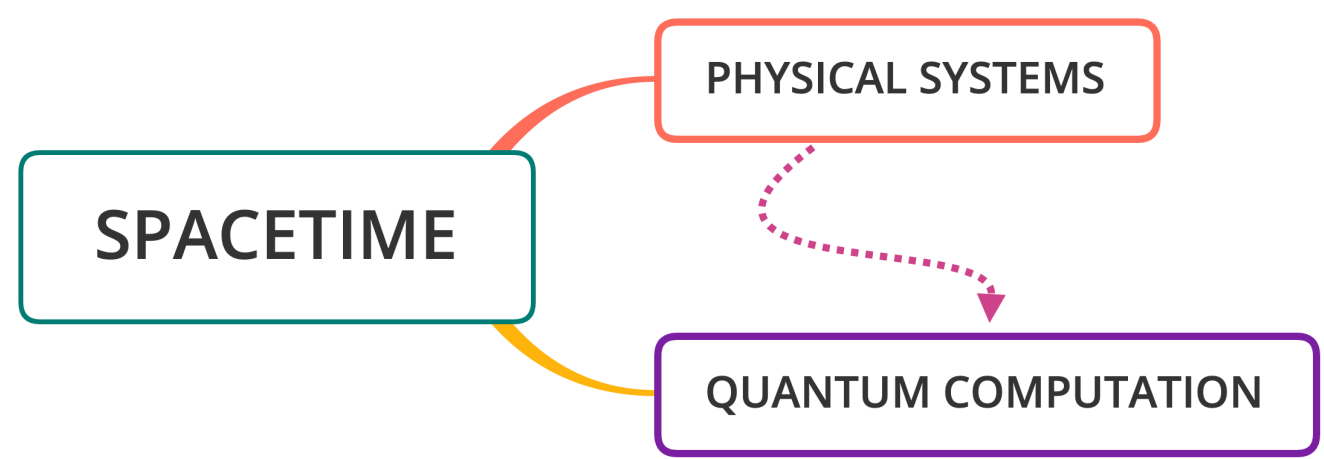

\section{Quantum Computation}

6. $[3,4]$

\section{Final Remarks}

7. If the conjecture (4) proves correct, then all physical systems are equivalent to simulations/circuits of a quantum computer [5].

\section{Open Invitation}

Review, add content, and co-author this paper $[6,7]$.

Join the Open Quantum Collaboration (https://bit.ly/ojmp-slack). Send your contribution to mplobo@uft.edu.br.

\section{Open Science}

The latex file for this paper together with other supplementary files are available [2]. 


\section{Ethical conduct of research}

This original work was pre-registered under the OSF Preprints [8], please cite it accordingly [9]. This will ensure that researches are conducted with integrity and intellectual honesty at all times and by all means.

\section{Acknowledgement}

+ Center for Open Science https://www.cos.io

+ Open Science Framework https://osf.io

\section{References}

[1] XMind. Mind mapping software. https://www.xmind.net/

[2] Lobo, Matheus P. "Open Journal of Mathematics and Physics (OJMP)." OSF, 21 Apr. 2020. https://doi.org/10.17605/osf.io/6hzyp

[3] Mermin, N. David. Quantum computer science: an introduction. Cambridge University Press, 2007.

[4] Nielsen, M. A., Chuang I. L. Quantum computation and quantum information. Cambridge University Press, 2010.

[5] Lobo, Matheus P. "Spacetime Is Entangled in a Bell State." OSF Preprints, 27 Nov. 2019. https://doi.org/10.31219/osf.io/xc4ys

[6] Lobo, Matheus P. "Microarticles." OSF Preprints, 28 Oct. 2019. https://doi.org/10.31219/osf.io/ejrct 
[7] Lobo, Matheus P. "Simple Guidelines for Authors: Open Journal of Mathematics and Physics." OSF Preprints, 15 Nov. 2019. https://doi.org/10.31219/osf.io/fk836

[8] COS. Open Science Framework. https://osf.io

[9] Lobo, Matheus P. "Spacetime Is a Quantum Computer." OSF Preprints, 21 June 2020. https://doi.org/10.31219/osf.io/rnckw

\section{The Open Quantum Collaboration}

Matheus Pereira Lobo (lead author, mplobo@uft.edu.br) $)^{1,2}$

https://orcid.org/0000-0003-4554-1372

${ }^{1}$ Federal University of Tocantins (Brazil)

${ }^{2}$ Universidade Aberta (UAb, Portugal) 